\title{
Structured risk assessment and violence in acute psychiatric wards: randomised controlled trial
}

\author{
Christoph Abderhalden, Ian Needham, Theo Dassen, Ruud Halfens, Hans-Joachim Haug \\ and Joachim E. Fischer
}

\section{Background}

There is a lack of research on the possible contribution of a structured risk assessment to the reduction of aggression in psychiatric in-patient care.

\section{Aims \\ To assess whether such risk assessments decrease the incidence of violence and coercion.}

\section{Method}

A cluster randomised controlled trial was conducted with 14 acute psychiatric admission wards as the units of randomisation, including a preference arm. The intervention comprised a standardised risk assessment following admission with mandatory evaluation of prevention in highrisk patients.

\section{Results}

Incidence rates decreased substantially in the intervention wards, whereas little change occurred in the control wards The adjusted risk ratios suggest a $41 \%$ reduction in severe aggressive incidents and a $27 \%$ decline in the use of coercive measures. The severity of aggressive incidents did not decrease.

\section{Conclusions}

Structured risk assessment during the first days of treatment may contribute to reduced violence and coercion in acute psychiatric wards.

\section{Declaration of interest}

None. Funding detailed in Acknowledgements.
Aggression and violence are a major problem in acute psychiatric wards, ${ }^{1,2}$ with career prevalence rates of being assaulted approximating $100 \%$ for mental healthcare staff. ${ }^{3}$ Manifest or impending violence is frequently managed by coercive measures such as seclusion, restraint or forced medication. Reducing the perceived need for coercion and the rate of aggressive incidents would advance the quality of psychiatric care. A prerequisite for adequate prevention is the assessment of high-risk situations. ${ }^{2}$ Previous research has related specific aspects of acute patient behaviour to subsequent violent outbursts. ${ }^{4,5}$ Building on this research, we developed a modified risk prediction scale. ${ }^{6}$ This study evaluates the effect of the routine use of this scale on the frequency and severity of patient aggression and the use of coercive measures in a multicentre trial.

\section{Method}

We conducted a prospective multicentre randomised waiting-list controlled trial with wards as the unit of randomisation and with the inclusion of a preference arm to assess the impact of a structured risk assessment on the incidence rate of severe patient aggression and coercive measures. Data collection and data verification procedures were pilot tested in an independent study involving two wards. ${ }^{7}$ The study was approved by six regional research ethics committees.

\section{Study area and eligibility of wards}

In the German-speaking part of Switzerland, 32 psychiatric hospitals provide psychiatric in-patient treatment for approximately $75 \%$ (5 376800 persons) of the Swiss population on 324 wards To be eligible for participation, a psychiatric ward had to meet the following criteria:
(a) the majority of patients had an acute psychiatric disorder
(b) patients were admitted directly onto the ward
(c) patients usually stayed less than 3 months on the ward

(d) patients were older than 18 years and younger than 65 years

(e) the ward admitted all potential patients and was not specialised for the treatment of specific disorders (e.g. depression, addiction).

\section{Eighty-six wards satisfied these criteria.}

\section{Recruitment and design}

The 86 acute wards were invited to partake in a large intervention trial, of which one arm was a structured risk assessment. Sixty-two wards declined to participate, including ten wards predominantly treating private patients with few involuntary admissions. Nineteen wards consented to be randomised within the trial, and five wards preferred to introduce the study protocol of structured risk assessment without randomisation. Randomisation was carried out prior to inclusion on the basis of a computer-generated random-number list. Here, we report on the four wards randomised to structured risk assessment, the five wards randomised to the waiting-list control arm, and the five wards of the preference group (Fig. 1). After enrolment, wards collected baseline data during a 3-month period (phase 1), followed by the 3-month intervention period (phase 2). The first ward was enrolled in June 2002 and the last ward completed the study in April 2004.

\section{Intervention}

The intervention consisted of a structured short-term risk assessment for every new patient during the first 3 days of hospitalisation. The instrument was the previously validated extended Swiss version of the Brøset Violence Checklist (BVC-CH) ${ }^{5,6,8}$ In principle, the $\mathrm{BVC}-\mathrm{CH}$ requires nurses to rate six patient behaviours (confusion, irritability, boisterousness, verbal threats, physical threats and attacks on objects) and to perform an overall subjective assessment of the risk of imminent violence using a slide-rule visual analogue scale. These combined ratings produce a score between 0 (very low risk) and 12 (high risk). The interrater 


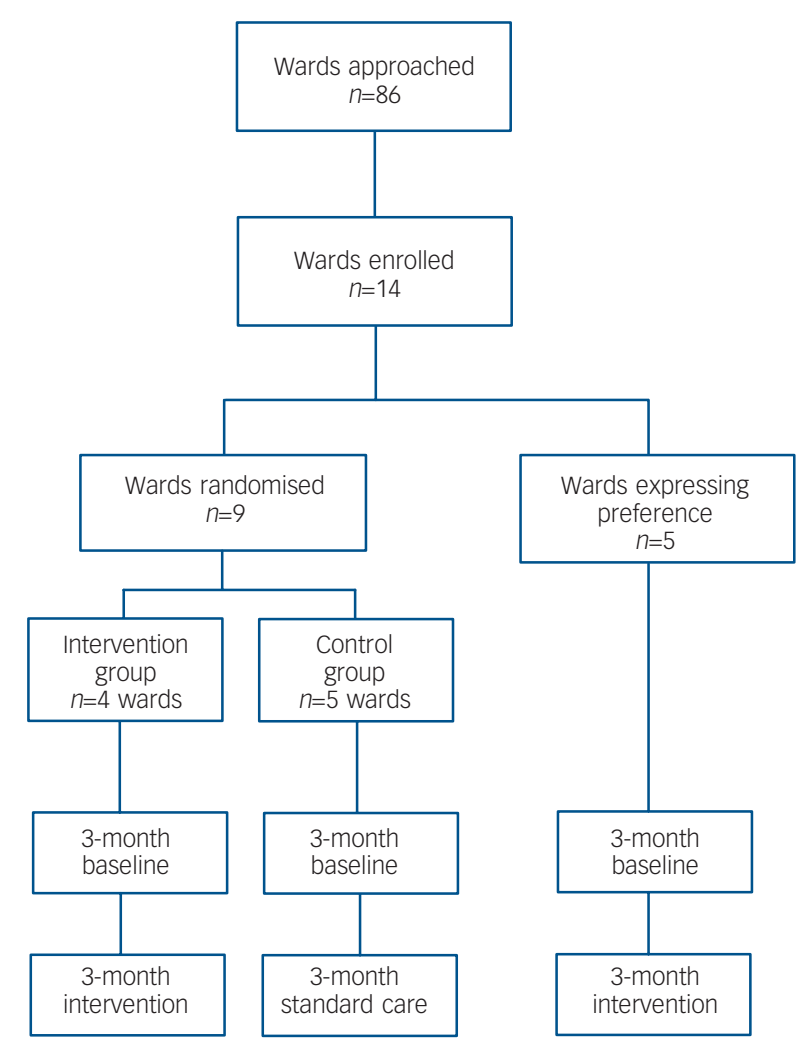

Fig. 1 Recruitment and follow-up. (to self, others or property). The severity of the incidents was measured using the SOAS-R scoring system, ranging from 0 to 22 points. Following the recommendation of the authors of the SOAS-R, incidents with a score of 9 or more points were regarded as severe (T. Palmstierna, personal communication, 2003). Coercive measures were recorded on a standardised form developed and pre-tested on the basis of existing formats in general use in the area. ${ }^{7}$ The form covered a wide range of measures, from forced injection of psychotropic medication to seclusion and mechanical restraint. For this study coercive measures were recoded into dichotomous data (present/absent).

\section{Data collection}

In order to control for possible recruitment bias, we conducted a survey of all wards within the study area prior to our investigation using a questionnaire enquiring about size of the wards, staffing and the facilities for managing aggression and violence. ${ }^{12}$ In addition, we asked the ward leaders to rate the severity of the problem and the resources for aggression management (Table 1). During both study periods, all aggressive incidents were registered using the SOAS-R form. Coercive measures were recorded on the purpose-designed study form. Physical attacks were considered if the SOAS-R description of the incident met both the following criteria:

(a) means of aggression: objects or dangerous objects or parts of the body

(b) target of aggression: a person other than the patient herself or himself.

To estimate possible underreporting of aggressive incidents, two investigators (C.A. and I.N.) regularly visited the study wards on randomly selected dates. During these site visits, all patient records were hand-reviewed for the preceding 3 days to detect any evidence of an aggressive incident. After termination of the study, each incident as abstracted from the patient records was compared with the available SOAS-R report forms. This made it possible to estimate the extent of underreporting and to assess the severity of those incidents for which no corresponding SOAS-R form was available. The same investigators abstracted additional patient data in a standardised format from the hospitals' databases. These data included admission and discharge dates, age, gender, type of admission (voluntary $v$. involuntary) and main ICD-10 psychiatric diagnosis. ${ }^{13}$

\section{Data analysis}

For each study period and ward, we calculated the incidence rate of events per 100 hospitalisation days. For this analysis, we included all aggressive incidents directed towards other persons or objects, but excluded pure aggression to self. The primary outcome was the rate of severe incidents with a SOAS-R score of 9 or more. Secondary outcomes were the rate of physical attacks and the rate of coercive measures. Ninety-five per cent confidence intervals for rates were calculated assuming independence of the probability of an incident for individual hospitalisation days (no correction for autocorrelation). From these raw incidence rates we calculated the risk ratios (RRs) for an event for each of the three study arms. The change in incidence rates between the intervention and control groups was tested using a test for the difference between two proportions (Statistica version 6). We used the number of patients not the number of treatment days for the calculation of the degrees of freedom. This is equivalent to the Geisser-Greenhouse lower-bound test for repeated-measures designs to control for sphericity. ${ }^{14}$ 
Owing to the non-normal distribution of data, comparisons between participating and non-participating wards were conducted using the Mann-Whitney $U$-test for independent samples and the chi-squared test or Fisher's exact test. Unless otherwise stated, data are reported as means and standard deviations. For all analyses, statistical significance was determined as a two-sided error probability of $\alpha=0.05$. Data were analysed using SPSS version 10.0 and Confidence Interval Analysis version 2.1 (University of Southampton, UK) for Windows.

\section{Results}

\section{Study population}

During both study periods, the 14 participating wards admitted a total of 2364 patients, accounting for 45913 hospitalisation days. The study wards had a mean number of beds of 16.9 (s.d.=1.9, range 13-19) and a mean number of staff (in full-time equivalents) of 0.71 (s.d.=0.2) per patient. Table 1 compares patient and ward characteristics, including information available for the non-participating wards. The data indicate similar ward characteristics, excluding ward managers' perception of aggression as a greater problem on the intervention wards. Also, the distribution of patient diagnoses across the intervention and the control arm of the study at baseline were comparable. In contrast, the preference arm had significantly fewer patients with ICD-10 F2 diagnoses (schizophrenia, schizotypal and delusional disorders): $26.5 \% \mathrm{v}$. $33.4 \%$ in the intervention wards and $35.7 \%$ in the control wards (Table 1).

Of the 2364 patients $(46.6 \%$ females; mean age $=39.5$ years, s.d. $=14.2$, range $14-95), 56 \%$ were admitted on a voluntary basis.
The involuntary admission rate of $44 \%$ is typical for wards within German-speaking Switzerland. The median length of stay was 9 days, with an average of 19 days (s.d.=26.8, range 1-265). The ICD-10 diagnoses of the patients were as follows: organic, including symptomatic, mental disorders (F0), $n=78$ (3.3\%); disorders due to psychoactive substance use (F1), $n=574$ (24.3\%); schizophrenia, schizotypal and delusional disorders (F2), $n=734$ (31.0\%); mood (affective) disorders (F3), $n=382$ (16.2\%); neurotic, stress-related and somatoform disorders, behavioural syndromes associated with physiological disturbances and physical factors (F4), $n=339$ (14.3\%); personality disorders of adult personality and behaviour (F6), $n=76$ (3.2\%); others (e.g. mental retardation, disorders of psychological development, behavioural and emotional disorders with onset occurring in childhood and adolescence, $n=66$ (2.8\%); and missing, $n=115$ (4.9\%).

\section{Aggressive incidents and coercive measures}

Over both phases of the study, 770 aggressive incidents were reported involving 314 patients (13.3\% of all patients) and 632 coercive measures were recorded. The difference between these numbers is attributable to the fact that not all aggressive incidents were followed by coercive measures. Additionally, coercive measures were sometimes employed to prevent aggression. Of the 770 aggressive incidents, 418 (54\%) had a SOAS-R score of 9 or above and 258 (34\%) incidents were physical attacks. The overall incidence rate of severe aggressive events during the baseline period was 1.09 (95\% CI $0.96-1.24)$ per 100 hospitalisation days. The overall incidence rate of coercion during baseline was 1.57 (95\% CI 1.41-175) per 100 hospitalisation days. Further rates are provided in Table 2.

\begin{tabular}{|c|c|c|c|c|c|}
\hline & $\begin{array}{l}\text { Intervention wards } \\
\qquad(n=4)\end{array}$ & $\begin{array}{l}\text { Preference wards } \\
\qquad(n=5)\end{array}$ & $\begin{array}{l}\text { Control wards } \\
\qquad(n=5)\end{array}$ & $\begin{array}{l}\text { Non-participating } \\
\text { wards }(n=68)^{\mathrm{a}}\end{array}$ & $P$ \\
\hline Number of beds: mean (s.d.) & $17.5(1.29)$ & $15.4(2.19)$ & $18.0(1.23)$ & $16.5(4.0)$ & $0.301^{b}$ \\
\hline Nursing staff per bed, FTE: mean (s.d.) & $0.67(0.24)$ & $0.75(0.23)$ & $0.73(0.08)$ & $0.74(0.25)$ & $0.977^{\mathrm{b}}$ \\
\hline Proportion of wards always open, $\%$ & 0 & 0 & 0 & 15 & $0.151^{\mathrm{c}}$ \\
\hline Proportion of wards with $\geqslant 1$ seclusion room, $\%$ & 100 & 100 & 100 & 82 & $0.894^{c}$ \\
\hline $\begin{array}{l}\text { Aggression rated as } \\
\text { No or very small problem, \% } \\
\text { Small or medium problem, \% } \\
\text { Big or very big problem, \% }\end{array}$ & $\begin{array}{r}0.0 \\
0.0 \\
100.0\end{array}$ & $\begin{array}{r}0.0 \\
60.0 \\
40.0\end{array}$ & $\begin{array}{l}0.0 \\
60.0 \\
40.0\end{array}$ & $\begin{array}{l}14.7 \\
52.9 \\
36.6\end{array}$ & $0.254^{c}$ \\
\hline $\begin{array}{l}\text { Resources for aggression management rated as } \\
\text { Sufficient, \% } \\
\text { Insufficient, \% }\end{array}$ & $\begin{array}{l}75.0 \\
25.0\end{array}$ & $\begin{array}{l}80.0 \\
20.0\end{array}$ & $\begin{array}{l}60.0 \\
40.0\end{array}$ & $\begin{array}{l}79.4 \\
20.6\end{array}$ & $0.760^{c}$ \\
\hline $\begin{array}{l}\text { Patient baseline data } \\
\text { Male, \% } \\
\text { Age, years: mean (s.d.) }\end{array}$ & $\begin{array}{c}54.4 \\
39.0(13.1)\end{array}$ & $\begin{array}{c}47.5 \\
41.7(15.9)\end{array}$ & $\begin{array}{c}55.2 \\
38.0(14.3)\end{array}$ & & $\begin{array}{l}0.048^{d} \\
0.321^{b}\end{array}$ \\
\hline Length of stay, days: mean (s.d.) & $20.7(29.9)$ & $16.9(25.8)$ & $18.1(26.5)$ & & $0.468^{\mathrm{b}}$ \\
\hline Involuntary admissions, \% & 41.4 & 36.9 & 38.4 & & $0.433^{\mathrm{d}}$ \\
\hline $\begin{array}{l}\text { Main diagnosis at discharge, \% } \\
\text { F0 } \\
\text { F1 } \\
\text { F2 } \\
\text { F3 } \\
\text { F4 } \\
\text { F6 } \\
\text { Other }\end{array}$ & $\begin{array}{r}3.8 \\
26.2 \\
33.4 \\
15.5 \\
14.3 \\
4.0 \\
2.7\end{array}$ & $\begin{array}{r}1.7 \\
27.0 \\
26.5 \\
21.4 \\
21.9 \\
1.4\end{array}$ & $\begin{array}{r}4.3 \\
24.2 \\
35.7 \\
15.3 \\
11.5 \\
5.0 \\
4.1\end{array}$ & & $<0.001^{\mathrm{d}}$ \\
\hline $\begin{array}{l}\text { FTE, full-time equivalent. } \\
\text { a. Missing data from four wards. } \\
\text { b. Kruskal-Wallis test. } \\
\text { c. Fisher's exact test. } \\
\text { d. Chi-squared test. }\end{array}$ & & & & & \\
\hline
\end{tabular}




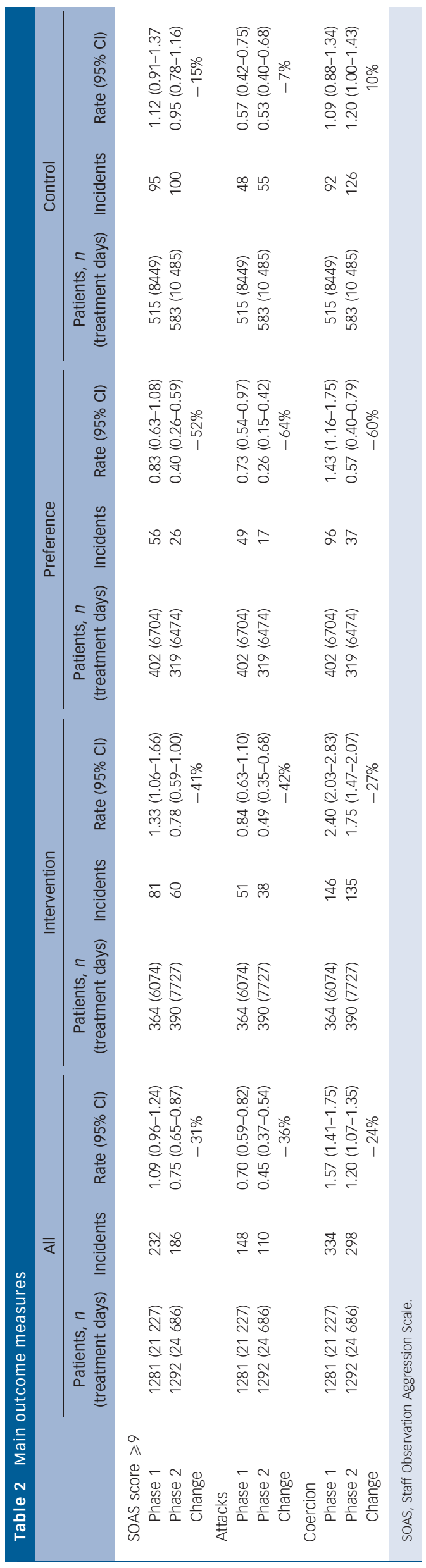

\section{Intervention}

From baseline to the intervention period, the rate of severe aggressive events with a SOAS-R score of 9 or more declined both in the control arm and in the intervention arm. The decline in the intervention wards ( $\mathrm{RR}=0.59,95 \% \mathrm{CI} 0.41-83$ ) was significantly larger $(P<0.001)$ than the decline in the control arms $(\mathrm{RR}=0.85,95 \% \mathrm{CI}$ 0.64-1.13). Raw and calculated data are presented in Table 2. Likewise, all rates declined more in the intervention wards compared with the control wards for all secondary outcomes: attacks $41 \% v$. $7 \%(P<0.001)$ and coercive measures $27 \% \mathrm{v}$. increase by $10 \%$ $(P<0.001)$. Finally, Fig. 2 illustrates that for all outcomes the effects were larger in the preference wards compared with the wards randomised to intervention or control. Similar results were

(a)
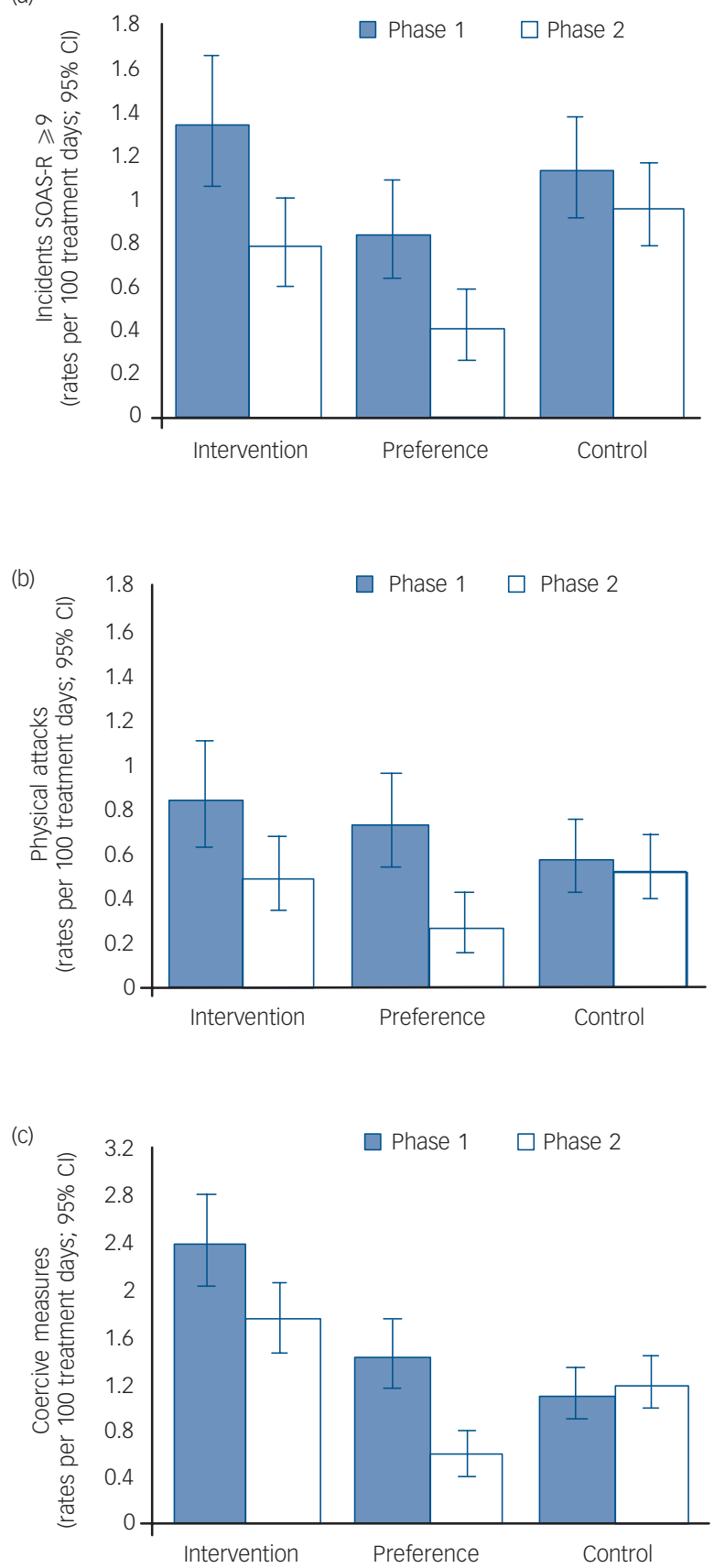

Fig. 2 Main outcome measures.

(a) Incidents with a Staff Observation Aggression Scale - Revised score of 9 or above; (b) physical attacks; (c) coercive measures. 
obtained when analysing the data as to the occurrence of days with any aggressive incident on the ward $v$. incident-free days (data not shown).

\section{Estimation of underreporting}

In contrast to the reduction in the rates of incidents and coercive measures, the mean severity of all recorded incidents increased in both the intervention group and the control group (Table 3). We had anticipated the possibility of an increasing underreporting of less severe events as the study progressed. Therefore, two investigators (C.A. and I.N) manually searched on randomly assigned dates the entire patient documentation and shift reports for 115 patients covering 460 treatment days. The search identified five incidents not registered with a corresponding SOAS-R form, with an estimated severity of the non-reported incidents ranging from 1 to 5 points. This suggests that predominantly minor incidents $(<9$ points) escaped reporting, whereas few severe incidents $(\geqslant 9$ points) remained unreported. Figure 3 compares the frequency of events stratified by score for each study period between the intervention and the control group.

\section{Discussion}

Using a structured assessment of the short-term risk of violence in acute psychiatric wards substantially reduced severe events of patient aggression (adjusted risk reduction 41\%) and also reduced the need for coercive measures (adjusted risk reduction 27\%). An even larger effect was observed in wards not consenting to randomisation but partaking in the study as a preference group. We consider a reduction in the incidence rates of aggression and coercion of the magnitude found in this study to be clinically relevant for patients and staff alike. The results are particularly encouraging because the intervention is relatively simple to implement, inexpensive and easy to integrate into daily practice. The study findings reflect the importance of continuing structured risk assessment, and support recommendations put forward in recent guidelines. ${ }^{2}$

\section{Mechanisms of effectiveness}

Strictly speaking, the intervention in this study consisted of three elements. First, there was the repeated structured determination of the risk in the first 3 days of each patient's hospital stay. Second, in cases of a high or very high risk, staff were encouraged to discuss preventive measures from a list provided on the risk assessment form. Third, in cases of very high risk, the teams were prompted to plan and implement preventive measures. Although related to each other, each of these elements might have been independently effective. For example, identification of patients with a high risk score allows allocating staff time spent during group meetings for discussion of preventive measures to a small subgroup of high-risk patients. Thus, there are several possible explanations for the observed overall effects. The obligation to assess all patients twice a day might have increased general awareness of potential dangers. This awareness itself might have fostered a more cautious approach and de-escalating staff behaviour. The risk assessment form might also have facilitated the intra- and interprofessional communication of risk. These factors, in combination with the obligatory discussion of high-risk situations, might have resulted in a more consistent team response to potentially dangerous patients. It is conceivable that the crucial factor might have been an improved intra- and interprofessional collaboration and unité de doctrine. Finally, part of the risk assessment form was a list of simple, practical prevention measures. The visibility of these measures might have acted as a constant reminder of possible ways to prevent escalation of aggressive situations or as a source of inspiration for aggression-related care planning. Taken together, the current intervention appears to be an example of a simple strategy to influence complex staff-patient interactions, in particular where patients are responsive to intervention. This is underscored by the finding that the intervention did not reduce the incidence rate of the severest aggressive events, which are probably not as amenable to staff interventions such as de-escalation. Although the systematic registering of aggression using the SOAS-R has been reported to be per se an effective mechanism to reduce aggression, ${ }^{15}$ we exclude this explanation given the stable aggression rates in the control wards.

Several design features of the study warrant discussion. In line with published research, we designed the study to combine risk assessment with elements of risk management, forgoing the possibility of delineating the effect of 'pure' risk assessment alone. We purposely included a preference arm in the study to simulate a patient preference randomised controlled trial paradigm or the comprehensive cohort design. ${ }^{16}$ In our study, the effect in the preference arm exceeded the effect observed in the group randomised to intervention. We offer several possible explanations for this. The patient population in the preference wards included a smaller proportion of people with a schizophrenic disorder; this raises the possibility that, beyond a preference effect, ${ }^{17}$ wards with fewer patients with schizophrenia may particularly benefit from the intervention. Alternatively, the more favourable results in the preference wards could also be seen as manifestation of staff characteristics and their preference for introducing risk assessment. This finding would then be independent of the patient population.

\section{Limitations}

In interpreting the findings of our study, several limitations must be considered. We relied on randomisation of wards to minimise bias. However, although the intervention and control wards were comparable with respect to most of the characteristics considered, we still had baseline differences in important aspects. All four of the intervention wards rated patient aggression as a big or very big problem, in contrast to two out of five control wards. Correspondingly, the baseline rates of aggression were higher on the intervention wards compared with the controls. This might have led to a higher sensitivity and perceived need to improve the situation, which might have increased the effect of the intervention. Because of the small number of wards, randomisation might not have been fully adequate to determine equality of the study groups. Additionally, a larger number of participating wards would have allowed the use of more sophisticated statistical models that simultaneously control for clustering and autocorrelation, i.e. a three-level model with days/events nested within

Table 3 Severity of aggressive incidents

\begin{tabular}{|c|c|c|c|c|c|c|c|c|c|}
\hline & \multicolumn{3}{|c|}{ Intervention } & \multicolumn{3}{|c|}{ Preference } & \multicolumn{3}{|c|}{ Control } \\
\hline & $n$ & Mean (s.d.) & $95 \% \mathrm{Cl}$ & $n$ & Mean (s.d.) & $95 \% \mathrm{Cl}$ & $n$ & Mean (s.d.) & $95 \% \mathrm{Cl}$ \\
\hline Phase 1 & 180 & 7.85 (4.5) & $7.2-8.5$ & 97 & $9.85(4.8)$ & $8.9-10.8$ & 185 & $8.14(4.7)$ & $7.5-8.8$ \\
\hline Phase 2 & 103 & $9.69(4.9)$ & $8.7-10.6$ & 33 & $12.79(4.8)$ & $11.1-14.5$ & 172 & $9.65(4.7)$ & $8.9-10.4$ \\
\hline
\end{tabular}



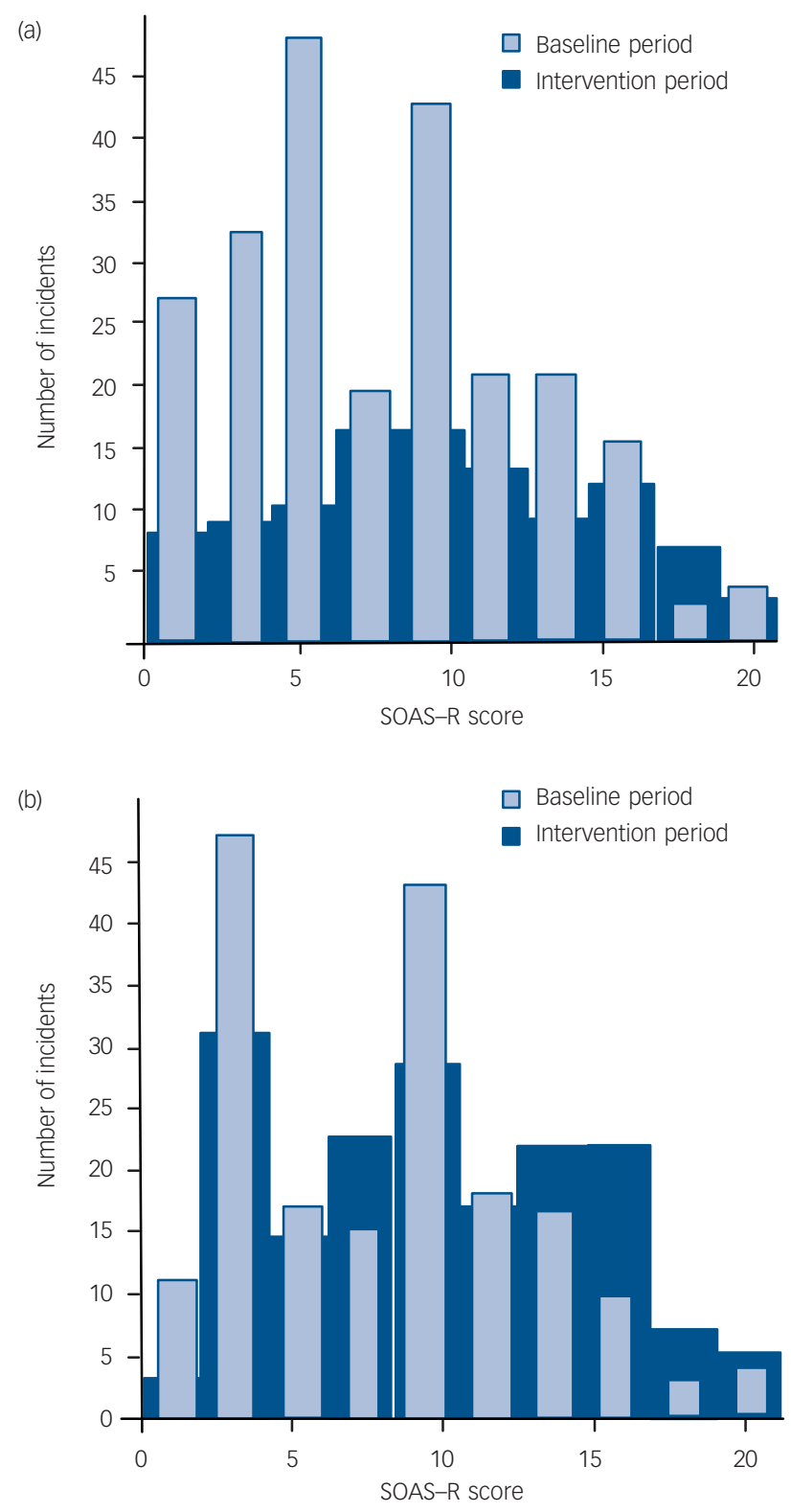

Fig. 3 Event severity across study periods.

SOAS-R, Staff Observation Aggression Scale - Revised. (a) intervention group; (b) control group. Each histogram shows the frequency of aggressive events standardised to the number of hospitalisation days during the intervention period. The comparison reveals that the intervention predominantly affected mild to moderately severe incidents; no reduction was seen for the most severe events.

patients nested within wards. Moreover, the nature of the intervention rendered masking impossible. A further limitation is the absence of data on the interventions actually implemented as a consequence of the risk assessment. Finally, data on the frequency of aggression or patient characteristics from the non-participating wards were unavailable. Caution is therefore warranted in generalising our data beyond the present study population. However, the aggression rates found in this study are comparable with rates found in other studies on psychiatric acute wards. ${ }^{11}$

\section{Clinical and practical implications}

Our finding that a simple and low-cost intervention, consisting of a risk assessment twice daily for the first 3 days of hospitalisation in acutely admitted psychiatric patients combined with a communication of risk scores as natural frequency numbers (e.g. 1 out of 10 patients instead of $10 \%, 1$ out of 1000 patients instead of $0.1 \%),{ }^{18}$ and a recommendation for action tailored to the risk level, reduced the incidence rate of coercive measures and severe aggressive incidents, suggesting that structured risk assessment may be a simple and cost-effective way of diminishing the problem of violent incidents in acute psychiatric wards.

Christoph Abderhalden, PhD, MNSc, Nursing and Social Education Research Unit, University of Bern Psychiatric Services, Berne, Switzerland; Ian Needham, PhD, MNSC, Clinic for Forensic Psychiatry, Centre of Psychiatry Rheinau, Rheinau, Switzerland; Theo Dassen, PhD, Department of Nursing Science, Humboldt University, Berlin, Germany; Ruud Halfens, PhD, Maastricht University, Maastricht, The Netherlands; Hans-Joachim Haug, MD, University of Zurich and Psychiatric Hospital Schloessli, Oetwil am See, Switzerland; Joachim E. Fischer, MD, MSc, Mannheim Institute of Public Health, Mannheim Medical Faculty, University of Mannheim Institute of Pl

Correspondence: Christoph Abderhalden, Nursing and Social Education Research Unit, University Bern Psychiatric Services, Bolligenstrasse 111، CH-3000 Berne 60, Switzerland. Email: Abderhalden@puk.unibe.ch

First received 22 Sep 2007, final revision 22 Jan 2008, accepted 1 Feb 2008

\section{Acknowledgements}

This study was supported by grant 3251B0-100710 from the swiss National Science Foundation. We gratefully acknowledge the help of the members of the European Violence in Psychiatry Research Group. We thank the staff of the participating wards for their collaboration in the collection of data. Finally, we acknowledge the statistical advice by Julian F. Thayer during revision of the manuscript.

\section{Appendix}

\section{Preventive measures for consideration in high-risk situations}

No specific measure to prevent an attack Careful observation

General conversation (directed to reduce aggression) Walk outdoors, one to one (directed to reduce aggression) Walk outdoors in a group (directed to reduce aggression) Reduction of demands (e.g. participation in activities)

Relaxation exercise

Confrontation with ward rules Discussion of risk with patient Talk down (to de-escalate)

Transfer to intensive area within ward

one-to-one observation for several hours Increase of medication dosage

Pro re nata medication given orally (psychotropic drugs) Open seclusion in the patient's own room (time out) Preventive seclusion (closed seclusion room) Injection of psychotropic drugs (forced/voluntary) Physical restraint (indicate number of points)

\section{References}

1 Whittington R, Richter D (eds). Violence in Mental Health Settings. Springer, 2006.

2 National Institute for Clinical Excellence. Violence: The Short-term Management of Disturbed/Violent Behaviour in In-patient Psychiatric Settings and Emergency Departments (Clinical Guideline 25). NICE, 2005.

3 Menckel E, Viitasara E. Threats and violence in Swedish care and welfare magnitude of the problem and impact on municipal personnel. Scand $J$ Caring SCi 2002; 16: 376-85.

4 Linaker OM, Busch Iversen, $\mathrm{H}$. Predictors of imminent violence in psychiatric inpatients. Acta Psychiatr Scand 1995; 92: 250-4.

5 Almvik R, Woods P. The Brøset Violence Checklist (BVC) and the prediction of inpatient violence: some preliminary results. Perspect Psychiatr Care 1998; 5: 208-11.

6 Abderhalden $\mathrm{C}$, Needham I, Dassen $\mathrm{T}$, Halfens $\mathrm{R}$, Haug $\mathrm{H}$, Fischer J. Predicting inpatient violence using an extended version of the 
Brøset-Violence-Checklist: instrument development and clinical application. BMC Psychiatry 2006; 6: 17.

7 Needham I, Abderhalden C, Meer R, Dassen T, Haug HJ, Halfens RJG Fischer JE. The effectiveness of two interventions in the management of patient violence in acute mental inpatient settings: report on a pilot study. J Psychiatr Ment Health Nurs 2004; 11: 595-601.

8 Almvik R, Woods P, Rasmussen K. The Brøset Violence Checklist: sensitivity, specificity and interrater reliability. $J$ Interpers Violence 2000; 15: 1284-96.

9 Abderhalden C, Gurtner C. Wie reliabel ist die Brøset-Gewalt-Checkliste (BVC) zur Einschätzung des kurzfristigen Gewaltrisikos? In Kompetenz - zwischen Qualifikation und Verantwortung (eds M Schulz, C Abderhalden, I Needham S Schoppmann, H Stefan): 105-9. Ibicura, 2007.

10 Nijman HL, Muris $\mathrm{P}$, Merckelbach HLGJ, Palmstierna T, Wistedt B, Vos AM Van Rixtel A, Allertz W. The Staff Observation Aggression Scale - Revised (SOAS-R). Aggress Behav 1999; 25: 197-209.

11 Nijman HL, Palmstierna T, Almvik R, Stolker JJ. Fifteen years of research with the Staff Observation Aggression Scale: a review. Acta Psychiatr Scand 2005 111: $12-21$
12 Needham I, Abderhalden C, Dassen T, Haug HJ, Fischer JE. Coercive procedures and facilities in Swiss psychiatry. Swiss Med Wkly 2002; 132 253-8.

13 World Health Organization. The ICD-10 Classification of Mental and Behavioural Disorders. Diagnostic Criteria for Research. WHO, 1992.

14 Turner JR, Thayer JF. Introduction to Analysis of Variance: Design, Analysis, and Interpretation. Sage, 2001

15 Nijman $\mathrm{H}$, Merckelbach $\mathrm{H}$, Evers $\mathrm{C}$, Palmstierna $\mathrm{T}$, à Campo J. Prediction of aggression on a locked psychiatric admissions ward. Acta Psychiatr Scand 2002; 105: 390-5

16 Brewin CR, Bradley C. Patient preferences and randomised clinical trials. BMJ 1989; 299: 13-5

17 Howard L, Thornicroft G. Patient preference randomised controlled trials in mental health research. Br J Psychiatry 2006; 188: 303-4.

18 Hoffrage $U$, Lindsey S, Hertwig R, Gigerenzer G. Medicine: communicating statistical information. Science 2000; 290: 2261-2.

\section{psychiatry} in pictures

\section{Egas Moniz (1875-1955), the father of psychosurgery}

Paolo Fusar-Poli, Paul Allen and Philip McGuire, from Neuroimaging Section, Department of Psychological Medicine, Institute of Psychiatry, Kings's College London, UK

In 1949 the Portuguese neurologist Antônio Caetano de Abreu Freire Egas Moniz was jointly awarded the Nobel Prize in medicine with the Swiss physiologist Walter Hess. At the 1935 International Neurological Conference in London, Moniz encountered the work of Fulton and Jacobsen who had observed behavioural changes in chimpanzees following removal of the frontal lobes. Together with Almeida Lima, Moniz initially adapted the technique for humans by drilling holes in the skull and injecting alcohol into the frontal lobes. The procedure of parietal prefrontal leucotomy was later developed, involving severing fibre tracts between the thalamus and the frontal lobes with a retractable wire loop or 'leucotome'. The American psychiatrist Walter Freeman further developed this by accessing the frontal lobes through the eye sockets (trans-orbital leucotomy or lobotomy). The procedure was eventually abandoned as a therapy for schizophrenia with the advent of the phenothiazines. Dr Egas Moniz became an invalid and retired (1945) after he was shot in the spine by one of his patients. He died in Lisbon in 1955.
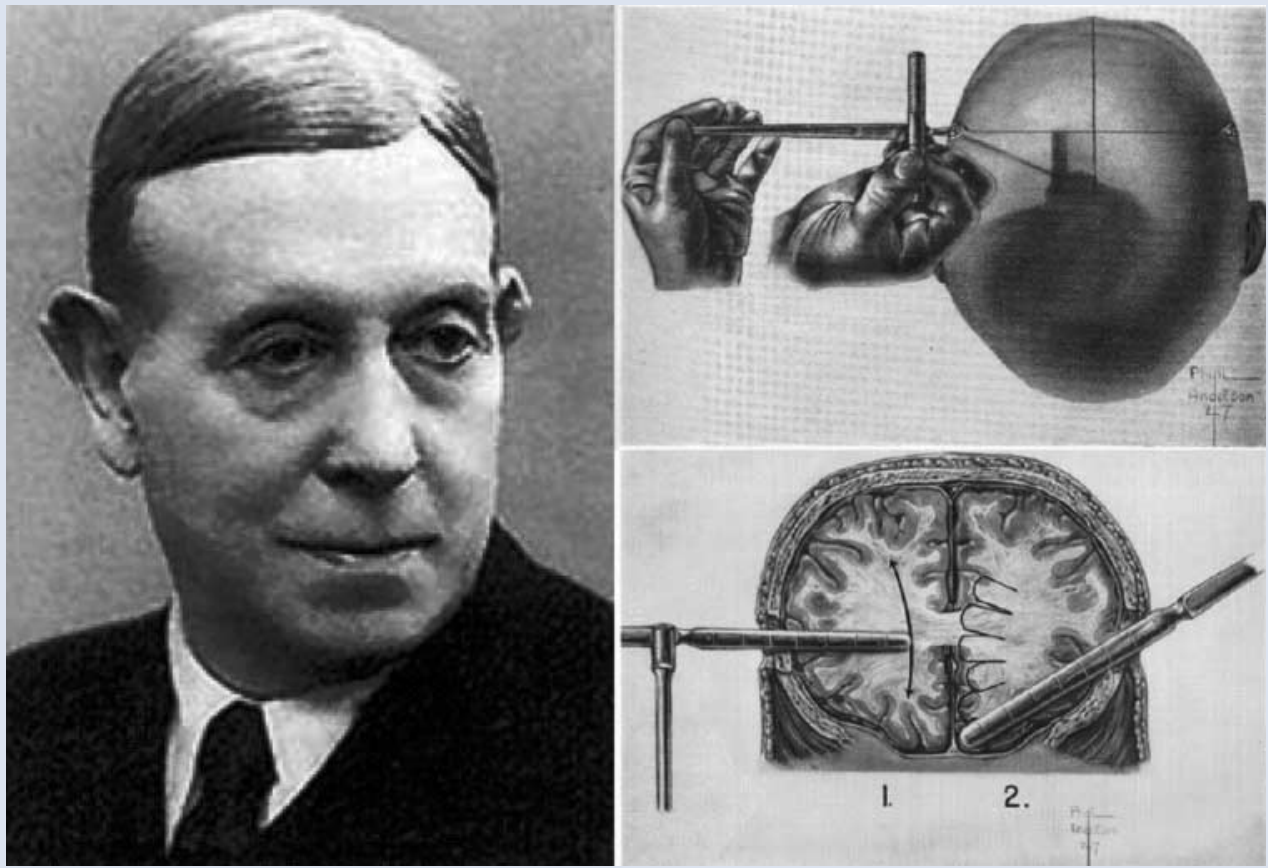

Antônio Caetano de Abreu Freire Egas Moniz father of psychosurgery.
Above: The surgeon is cutting the pathways in the left upper quadrant with a sweeping incision using the precision leucotome.

Below: (1) Sweeping incisions made with the precision leucotome are (2) deepened with a wider blunt knife - the radial stab incisor. 